\title{
Binomial Coefficients and Triangular Numbers
}

\author{
Kantaphon Kuhapatanakul and Anthony G. Shannon
}

\begin{abstract}
We produce formulas of sums the product of the binomial coefficients and triangular numbers. And we apply our formula to prove an identity of Wang and Zhang. Further, we provide an analogue of our identity for the alternating sums.
\end{abstract}

Index Terms - binomial coefficient; triangular number.

Mathematics Subject Classification: 11B65.

\section{INTRODUCTION}

The triangular number $T_{n}$ is a number obtained by adding all positive integers less than or equal to a given positive integer $n$,

$$
T_{n}=\frac{n(n+1)}{2}
$$

It is also the binomial coefficient

$$
T_{n}=\left(\begin{array}{c}
n+1 \\
2
\end{array}\right)
$$

which counts the number of distinct pairs to be selected from $n+1$ objects.

The Fibonacci numbers $F_{n}$ are defined by

$$
F_{0}=0, F_{1}=1 \text { and } F_{n+1}=F_{n}+F_{n-1}(n \geq 1) .
$$

The triangular numbers and Fibonacci numbers, even today, are fascinating to mathematicians (young or old), and have many interesting properties and applications to almost every fields of science and art (e.g., see [2], [3]).

Some identities of the sums of product of the binomial coefficients and Fibonacci numbers $F_{n}$ (see [4]) are

$$
\begin{aligned}
& \sum_{i=0}^{n}\left(\begin{array}{c}
n \\
i
\end{array}\right) F_{i}=1^{n} F_{2 n} \\
& \sum_{i=0}^{n}\left(\begin{array}{c}
n \\
i
\end{array}\right) F_{3 i}=2^{n} F_{2 n} \\
& \sum_{i=0}^{n}\left(\begin{array}{l}
n \\
i
\end{array}\right) F_{4 i}=3^{n} F_{2 n} .
\end{aligned}
$$

In this work, we first obtain analogous formulas for the triangular numbers. Next, we apply our formula to prove an identity of Wang and Zhang [8]. While the connections

Published on July 05, 2021.

K. Kuhapatanakul, Department of Mathematics, Faculty of Science, Kasetsart University, Bangkok, Thailand.

(corresponding e-mail: fscikpkk@ku.ac.th) between triangular and pentagonal numbers are well known (e.g., [6]) we also show a connection with geometric numbers in general.

\section{TRIANGULAR NUMBERS}

Throughout this paper, the symbol $\left(\begin{array}{l}n \\ k\end{array}\right)$ is the binomial coefficient, that is,

$$
\left(\begin{array}{l}
n \\
k
\end{array}\right)=\left\{\begin{array}{cl}
\frac{n !}{k !(n-k) !} & , n \geq k \\
0 & , n<k
\end{array} \text { and }\left(\begin{array}{l}
0 \\
0
\end{array}\right)=1 .\right.
$$

The Pascal's identity is

$$
\left(\begin{array}{c}
n+1 \\
i
\end{array}\right)=\left(\begin{array}{c}
n \\
i
\end{array}\right)+\left(\begin{array}{c}
n \\
i-1
\end{array}\right)
$$

Theorem 1 Let $T_{n}$ be the $n^{\text {th }}$ triangular number. For any positive integer $k$, we have

$$
\sum_{i=1}^{n}\left(\begin{array}{c}
n \\
i
\end{array}\right) T_{k i}=k n(k n+k+2) 2^{n-3}
$$

Proof. By induction on $n$, we see that identity (1) holds for $n$ $=1$. Now assume identity (1) is true for all integers $n \geq 1$. By Pascal's identity, the inductive hypothesis and the definition of triangular numbers, we obtain

$$
\begin{aligned}
\sum_{i=1}^{n+1}\left(\begin{array}{c}
n+1 \\
i
\end{array}\right) T_{k i}=\sum_{i=1}^{n+1}\left[\left(\begin{array}{c}
n \\
i
\end{array}\right)+\left(\begin{array}{c}
n \\
i-1
\end{array}\right)\right] T_{k i} \\
=\sum_{i=1}^{n}\left(\begin{array}{l}
n \\
i
\end{array}\right) T_{k i}+T_{k}+\sum_{i=1}^{n}\left(\begin{array}{c}
n \\
i
\end{array}\right)\left(T_{k i}+\sum_{j=1}^{k}(k i+j)\right) \\
=2 \sum_{i=1}^{n}\left(\begin{array}{c}
n \\
i
\end{array}\right) T_{k i}+T_{k}+\sum_{i=1}^{n}\left(\begin{array}{c}
n \\
i
\end{array}\right)\left(k^{2} i+T_{k}\right) \\
=2 k n(k n+k+2) 2^{n-3}+\frac{k(k+1)}{2} \\
+k^{2} n 2^{n-1}+\frac{k(k+1)\left(2^{n}-1\right)}{2}
\end{aligned}
$$

A. G. Shannon, Warrane College, University of New South Wales, Kensington, NSW 2033, Australia.

(e-mail: t.shannon@warrane.unsw.edu.au) 


$$
\begin{aligned}
& =k n(k n+k+2) 2^{n-2}+2 k^{2} n 2^{n-2}+2 k(k+1) 2^{n-2} \\
= & k(n+1)(k(n+1)+k+2) 2^{n-2},
\end{aligned}
$$

which shows that (1) holds for $n+1$, thereby proving the theorem.

From Theorem 1, we obtain the following examples for $k=1,2,3,4$.

$$
\begin{aligned}
& \text { (i) } \sum_{i=1}^{n}\left(\begin{array}{c}
n \\
i
\end{array}\right) T_{i}=n(n+3) 2^{n-3} \\
& \text { (ii) } \sum_{i=1}^{n}\left(\begin{array}{c}
n \\
i
\end{array}\right) T_{2 i}=2 n(2 n+4) 2^{n-3} \\
& \text { (iii) } \sum_{i=1}^{n}\left(\begin{array}{c}
n \\
i
\end{array}\right) T_{3 i}=3 n(3 n+5) 2^{n-3} \\
& \text { (iv) } \sum_{i=1}^{n}\left(\begin{array}{l}
n \\
i
\end{array}\right) T_{4 i}=4 n(4 n+6) 2^{n-3} .
\end{aligned}
$$

These lead to a variety of other results; for example, when $n=3$, we get

$$
\sum_{i=1}^{3}\left(\begin{array}{l}
3 \\
i
\end{array}\right) T_{k i}=6 T_{2 k}=3 a_{k}
$$

in which $\left\{a_{k}\right\} \equiv\{6,20,42,72,110, \ldots\}$, where $a_{n}$ is the number of edges in an $(n+1) \times(n+1)$ square grid with all horizontal, vertical and diagonal segments filled in (see A002943 in [7]). Clearly,

$$
a_{n}=2 n(2 n+1)=2\left(\begin{array}{c}
2 n+1 \\
2
\end{array}\right)
$$

Next, we produce an alternating sum of the product of the binomial coefficients and triangular numbers. The following identities are easily verified

$$
\sum_{i=0}^{n}(-1)^{i}\left(\begin{array}{l}
n \\
i
\end{array}\right)=0 \quad \text { and } \sum_{i=0}^{n}(-1)^{i} i\left(\begin{array}{l}
n \\
i
\end{array}\right)=0 .
$$

Theorem 2 Let $n, k$ be two positive integers. We have

$$
\sum_{i=1}^{n}(-1)^{i}\left(\begin{array}{c}
n \\
i
\end{array}\right) T_{k i}=0
$$

Proof. By induction on $n$, we see that identity (2) holds for $n$ $=1$. Now assume identity (2) is true for all integers $n \geq 1$. By Pascal's identity, inductive hypothesis and definition of triangular numbers, we obtain

$$
\sum_{i=1}^{n+1}(-1)^{i}\left(\begin{array}{c}
n+1 \\
i
\end{array}\right) T_{k i}=\sum_{i=1}^{n+1}(-1)^{i}\left[\left(\begin{array}{c}
n \\
i
\end{array}\right)+\left(\begin{array}{c}
n \\
i-1
\end{array}\right)\right] T_{k i}
$$

$$
\begin{aligned}
& =\sum_{i=1}^{n+1}(-1)^{i}\left(\begin{array}{c}
n \\
i
\end{array}\right) T_{k i}+\sum_{i=1}^{n}(-1)^{i+1}\left(\begin{array}{c}
n \\
i
\end{array}\right)\left(T_{k i}+\sum_{j=1}^{k}(k i+j)\right) \\
& =-T_{k}+\sum_{i=1}^{n}(-1)^{i+1}\left(\begin{array}{c}
n \\
i
\end{array}\right)\left(k^{2} i+T_{k}\right) \\
& =-T_{k}+k^{2} \sum_{i=1}^{n}(-1)^{i+1} i\left(\begin{array}{c}
n \\
i
\end{array}\right)+T_{k} \sum_{i=1}^{n}(-1)^{i+1}\left(\begin{array}{c}
n \\
i
\end{array}\right) \\
& =-T_{k}+T_{k}=0,
\end{aligned}
$$

which shows that (2) holds for $n+1$, thereby proving the theorem.

\section{BINOMIAL COEFFICIENTS}

For fixed positive integer $m$, denote $A_{m, i}$ as the partial sum of binomial coefficients $\left(\begin{array}{c}m \\ j\end{array}\right)$ for $0 \leq j \leq i$, that is,

$$
A_{m, i}=\sum_{j=0}^{i}\left(\begin{array}{c}
m \\
j
\end{array}\right)=\left(\begin{array}{c}
m \\
0
\end{array}\right)+\left(\begin{array}{c}
m \\
1
\end{array}\right)+\left(\begin{array}{c}
m \\
2
\end{array}\right)+\cdots+\left(\begin{array}{c}
m \\
i
\end{array}\right) .
$$

It is easy to see that $A_{m, m}=2^{m}$ and $A_{m, k}+A_{m, m-k-1}=2^{m}$ for $0 \leq k \leq m$.

For a positive integer $n$, observe that

$$
\begin{aligned}
\sum_{i=0}^{n} A_{n, i} & =A_{n, 0}+A_{n, 1}+A_{n, 2}+\cdots+A_{n, n} \\
& =\frac{1}{2}\left(\left(A_{n, 0}+A_{n, n-1}\right)+\cdots+\left(A_{n, n-1}+A_{n, 0}\right)\right)+A_{n, n} \\
& =\frac{1}{2} n \cdot 2^{n}+2^{n} \\
& =(n+2) 2^{n-1} .
\end{aligned}
$$

We then obtain that

$$
\sum_{i=1}^{n}\left(\begin{array}{c}
n \\
i
\end{array}\right) T_{2 i}=n \sum_{i=0}^{n} A_{n, i}
$$

Moreover, Calkin [1] developed some curious identity of sums of 3-powers of the partial sum of binomial coefficients:

$$
\sum_{i=0}^{n} A_{n, i}^{3}=(n+2) 2^{3 n-1}-3 n \cdot 2^{n-2}\left(\begin{array}{c}
2 n \\
n
\end{array}\right) .
$$

Hirschhorn [5] obtained some recurrence relations of sums of powers of the partial sum of binomial coefficients and derived the identities involving first, second and third powers. Next, Wang and Zhang [8] generalized these identities involving first, second and third powers, one of these generalization is the following 


$$
\sum_{i=0}^{n} i A_{n, i}=n(3 n+5) 2^{n-3}
$$

Next, we shall prove identity (3) by using Theorem 2 .

Theorem 2 Let $n$ be a positive integers and $A_{n, i}$ as defined above. Then

$$
\sum_{i=0}^{n} i A_{n, i}=n(3 n+5) 2^{n-3}
$$

Proof. Consider

$$
\begin{aligned}
\sum_{i=0}^{n}(n-i) A_{n, i} & =\sum_{i=0}^{n}(n-i) \sum_{j=0}^{m}\left(\begin{array}{c}
m \\
j
\end{array}\right) \\
& =\sum_{i=0}^{n}\left(\begin{array}{l}
n \\
i
\end{array}\right) \sum_{j=1}^{n-i} j \\
& =\sum_{i=0}^{n}\left(\begin{array}{l}
n \\
i
\end{array}\right) T_{n-i} \\
& =\sum_{i=0}^{n}\left(\begin{array}{l}
n \\
i
\end{array}\right) T_{i} .
\end{aligned}
$$

Since

$$
\sum_{i=0}^{n}\left(\begin{array}{l}
n \\
i
\end{array}\right) T_{i}=n(n+3) 2^{n-3},
$$

we have

$$
\sum_{i=0}^{n}(n-i) A_{n, i}=n(n+3) 2^{n-3}
$$

Using the identity

$$
\sum_{i=0}^{n} A_{n, i}=(n+3) 2^{n-1}
$$

we get that

$$
\begin{aligned}
\sum_{i=0}^{n} i A_{n, i} & =\sum_{i=0}^{n} n A_{n, i}-\sum_{i=0}^{n}(n-i) A_{n, i} \\
& =n(n+2) 2^{n-1}-n(n+3) 2^{n-3} \\
& =n(3 n+5) 2^{n-3}
\end{aligned}
$$

as desired.

Corollary 3 For a positive integer $n$,

$$
\sum_{i=1}^{n}\left(\begin{array}{c}
n \\
i
\end{array}\right) T_{3 i}=3 \sum_{i=0}^{n} i A_{n, i} .
$$

\section{CONCLUSION}

The triangular numbers are linked to other geometric numbers through binomial identities. Thus, for the sequence of pentagonal numbers (see A000292 in [7]),

$$
\left\{p_{n}\right\} \equiv\{1,5,12,22,35,51,70, \ldots\}
$$

or

$$
p_{n}=\frac{3 n-1}{n+1}\left(\begin{array}{c}
n+1 \\
2
\end{array}\right)=\frac{3 n-1}{n+1} T_{n},
$$

we have the known

$$
T_{n}=\frac{1}{n} \sum_{i=1}^{n} p_{n}
$$

There are many other connections between the triangular and pentagonal numbers [6] as well as with other geometric numbers, such as the triangular pyramidal numbers $t_{n}$ (see A000292 in OEIS),

$$
\left\{t_{n}\right\} \equiv\{1,4,10,20,35,56,84, \ldots\}
$$

or

$$
t_{n}=p_{n-1}+F_{n+2} \quad(n>1),
$$

which are in turn connected with the triangular numbers by

$$
t_{n}=\sum_{i=1}^{n} T_{n}=\left(\begin{array}{c}
n+2 \\
3
\end{array}\right)
$$

\section{ACKNOWLEDGMENT}

The authors greatly appreciate the anonymous referee for his/her careful reading and helpful comments.

\section{REFERENCES}

[1] N. J. Calkin, "A curious binomial identities," Discrete Mathematics, vol. 131, pp. 335-337, 1994

[2] L. Depnarth, "A short history of the Fibonacci and golden numbers with their applications," Math. Educ. Sci. Technol., vol. 42, pp. 337367, 2011.

[3] T. Koshy, Fibonacci and Lucas numbers with applications, New York, NY: John Wiley and Sons; 2001.

[4] V. E. Hoggatt, Jr., "Some special Fibonacci and Lucas generating functions," The Fibonacci Quarterly, vol. 19, no. 2, pp. 121-138, 1971

[5] M. Hirschhorn, "Calkin's binomial identity," Discrete Mathematics, vol. 159, pp. 273-278, 1996.

[6] J. V. Leyendekkers and A. G. Shannon, "Integer structure analysis of odd powered triples: The significance of triangular versus pentagonal number," Notes on Number Theory and Discrete Mathematics, vol. 16 no. 4, pp. 6-13, 2010.

[7] N. J. A. Sloane, A Handbook of Integer Sequences, San Diego, CA: Academic Press, 1964. [The On-Line Encyclopedia of Integer Sequences oeis.org (OEIS)]

[8] J. Wang and Z. Zhang, "On extensions of Calkin's binomial identities," Discrete Mathematics, vol. 274, pp. 331-342, 2004. 